\title{
THE CUTTING PROPERTIES OF KUNZITE
}

\author{
By John L. Ramsey
}

In the process of cutting kunzite, a lapidary comes face-to-face with problem properties that sometimes remain hidden from the jeweler. By way of examining these problems, we present the example of a one-kilo kunzite crystal being cut. The cutting problems shown give ample warning to the jeweler to take care in working with kunzite and demonstrate the necessity of cautioning customers to avoid shocking the stone when they wear it in jewelry.

The difficulties inherent in faceting kunzite are legendary to the lapidary. Yet the special cutting problems of this stone also provide an important perspective for the jeweler. The cutting process reveals all of the stone's intrinsic mineralogical problems to anyone who works with it, whether cutter or jeweler. Knowledge of these problems also aids the person marketing the stone, both in conveying the specialness of the faceted gem and in advising customers of the proper care that it requires.

To explain the various problems presented by kunzite, this article describes the cutting of an extremely large kunzite crystal. In some ways, the size of the crystal magnifies the problems and renders them examinable in a manner that allows us to generalize to all kunzites.

It should first be mentioned that the availability of a one-kilo, essentially clean kunzite crystal from Brazil in today's marketplace is in itself a phenomenon. Certainly, a number of fine crystals from Brazil were available in the late 1950s and early 1960s, but these disappeared from the market some time ago. Afghanistan produces kunzite, but nothing of the size and quality of the crystal described. This particular crystal, which was virtually unflawed and of superb color (figure 1), had been held in a European collection for 25 years before its release to the author.

\section{THE PROBLEMS OF PERFECT CLEAVAGE AND RESISTANCE TO ABRASION}

Kunzite is a variety of spodumene, a lithium aluminum silicate (see box). Those readers who are familiar with either cutting or mounting stones in jewelry are aware of the problems that spodumene, in this case kunzite, invariably poses. For those unfamiliar, it is important to note that kunzite has two distinct cleavages. Perfect cleavage in a stone means that splitting, when it occurs, tends to produce plane surfaces. Cleavage in two directions means that the splitting can occur in a plane along either of two directions in the crystal. The property of cleavage, while not desirable in a gemstone, does not in and of itself mean trouble. For instance, diamond tends to cleave but splits with such difficulty that diamonds are cut, mounted, and worn with little trepidation. In contrast, the abrasive process used in sawing and faceting represents a danger with kunzite. The separation in kunzite happens very easily, especially if the stone is ground against the direction of cleavage, and it may simply fall apart during faceting.

To compound the problem, kunzite has an unusual resistance to abrasion. While the hardness of spodumene is $6 \frac{1 / 2}{2}$ to 7 , it grinds with more difficulty than corundum which, at 9, is exceeded in hardness only by diamond. The resistance no doubt comes from the crystal structure, which is also responsible for the problems with cleavage. The combination of these two problems in one gem material forces the cutter to delicately balance two factors: (1) a stone that resists abrasion

\section{ABOUT THE AUTHOR}

Mr. Ramsey is president of Ramsey Gem Import, Inc., Escondido, CA.

1982 Gemological Institule of America 


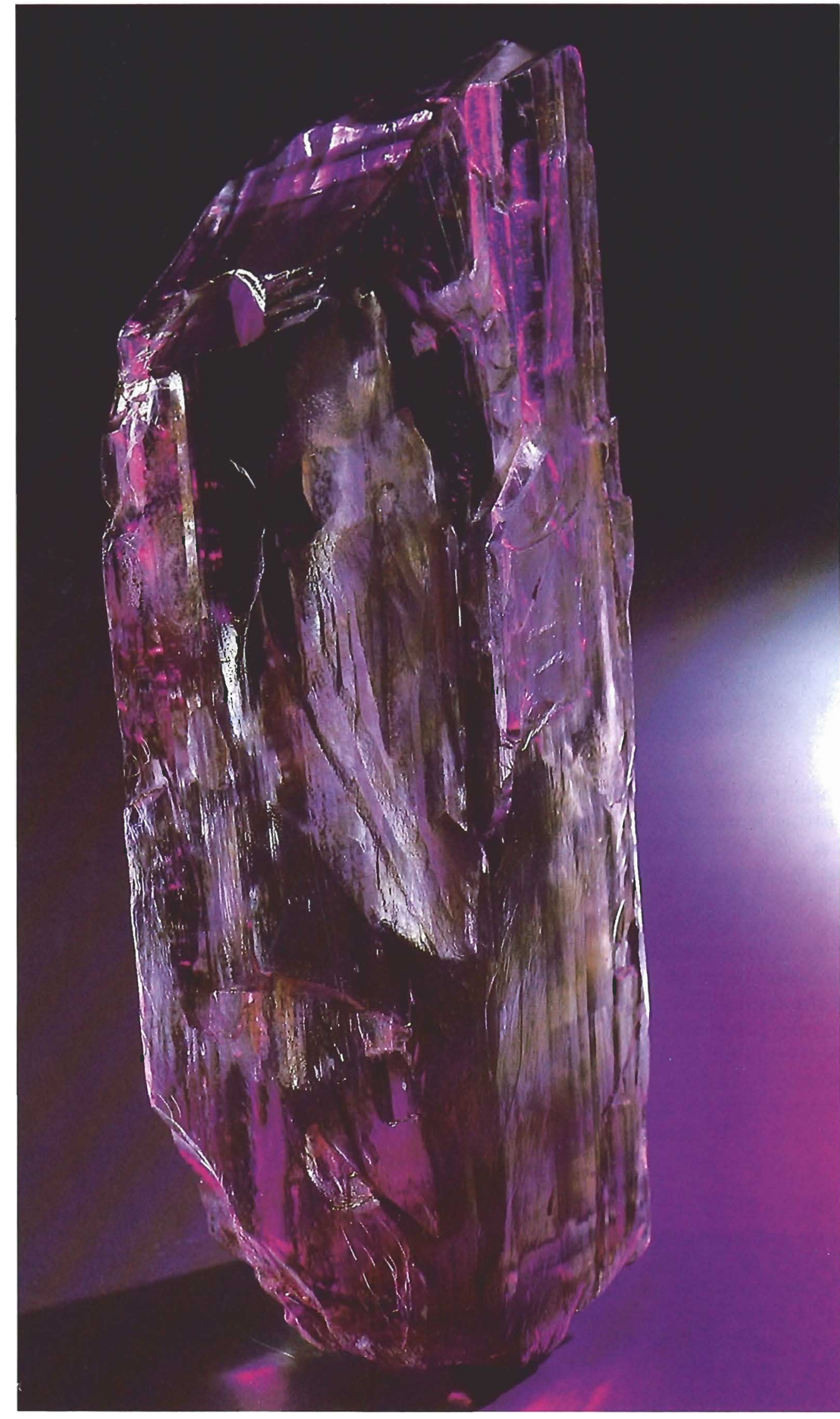

Figure 1. The one-kilo kunzite before cutting. The dimensions were $150 \mathrm{~cm} \times 5.1 \mathrm{~cm} \times$ $4.9 \mathrm{~cm}$. Photograph (1981 Laura Ramsey. 


\section{PROPERTIES OF SPODUMENE}

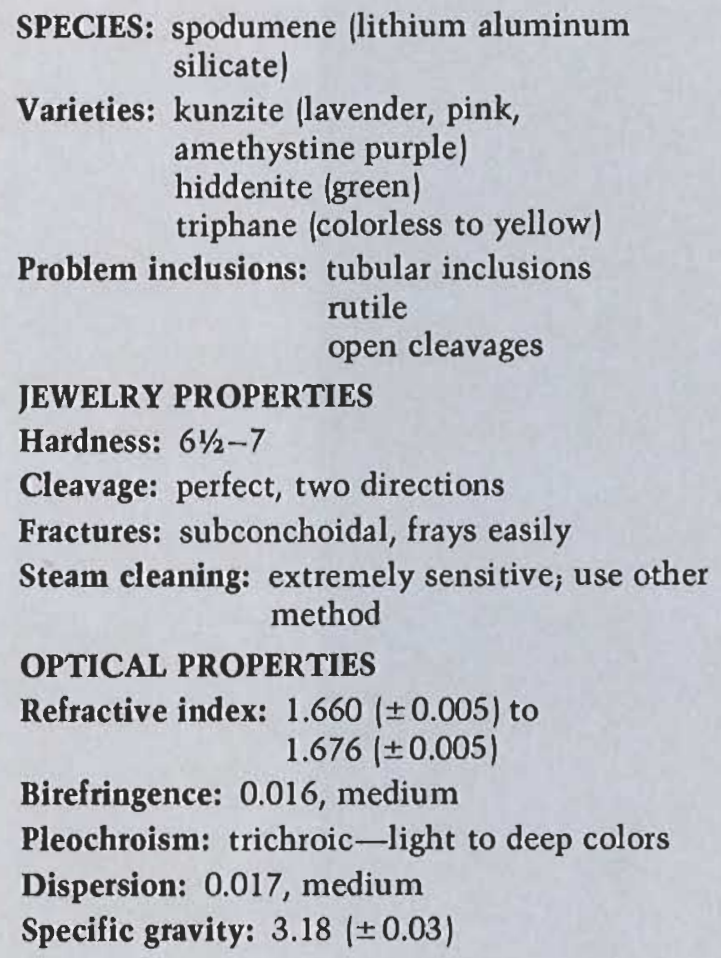

SPECIES: spodumene (lithium aluminum silicate)

Varieties: kunzite (lavender, pink, amethystine purple) hiddenite (green) triphane (colorless to yellow)

Problem inclusions: tubular inclusions rutile open cleavages

\section{JEWELRY PROPERTIES}

Hardness: $61 / 2-7$

Cleavage: perfect, two directions

Fractures: subconchoidal, frays easily

Steam cleaning: extremely sensitive; use other method

OPTICAL PROPERTIES

Refractive index: $1.660( \pm 0.005)$ to

$1.676( \pm 0.005)$

Birefringence: 0.016 , medium

Pleochroism: trichroic-light to deep colors

Dispersion: 0.017 , medium

Specific gravity: $3.18( \pm 0.03)$

must be given a fair amount of physical pressure against the diamond cutting wheel or it will not cut, and (2) a stone that will split easily in two directions may separate if too much pressure is placed against the lap.

In addition, the propensity of kunzite to fall apart during cutting tends to be aggravated by the fact that resistance to abrasion varies with respect to the different axes of the crystal. Since dopping is not an exact process, the cutter cannot determine precisely whether grinding will be only mildly difficult or extremely difficult. Kunzite never grinds easily. One positive point, though, is that kunzite is perhaps one of the easiest stones to polish.

\section{HOW LARGE A STONE TO CUT?}

With these factors in mind, the next step was to determine the optimum size of the finished material. The rule of thumb in cutting is usually to produce the largest gem possible from each piece of rough regardless of the species. This is done because large stones are relatively rare. For every one-carat diamond there are hundreds or thousands of 10 pointers, and so on through most of the known gem species.

Upon evaluating this kunzite, we saw that we had three cutting options: (1) one 1,500-ct. stone, (2) many smaller gems, or (3) several extremely large gems.

Cutting a 1,500-ct. stone was a temptation, since at that size it would have been one of the largest spodumenes ever faceted /see Sinkankas, 1959 , for a description of the cutting of an 1,800ct. green spodumene). Kunzite is distinctly trichroic, however, and the best color is seen parallel to the c-axis of the crystal, which is invariably also the narrow axis. While trichroism in kunzite varies from crystal to crystal, in this case the exceptionally high trichroism of the stone virtually dictated the cutting orientation: the table(s) had to lay perpendicular to the c-axis. The choice of cutting a single 1,500-ct. stone was, therefore, ruled out because of the poor color orientation that would have resulted.

Following the premise that in any gem the size/quality ratio is of paramount importance, we also ruled out the second alternative, that of cutting the crystal into many small stones /although we were assured by many gem dealers that there is a ready market for these smaller gems). Instead, we chose as our model the 800-plus-carat kunzite housed in the Smithsonian Institution, Washington, DC, which is one of the few large spodumenes of the variety kunzite with exceptional color and clarity.

From the point of view of aesthetics, there was only one choice. Not only did the trichroism of this crystal dictate the cutting preference, but the opportunity to cut some genuinely fine, large kunzite gems, and thus add to the other large stones already in existence, also prompted the selection of the third option.

\section{CUTTING THE CRYSTAL}

Tom Carleton, of Menasha, Wisconsin, agreed to do the cutting under the author's supervision. At this point, we looked to the existing literature for tips on cutting spodumene, but found little helpful information. The article by Sinkankas referred to earlier discusses some basic techniques, but new technology has contributed much to the field in the two decades since that article appeared. For example, whereas Sinkankas had to charge his 
own cutting laps, we had precharged, bonded and plated diamond laps. Then, too, there is some published information with which we disagree on the basis of previous experience cutting smaller spodumenes. In their book, Faceting for Amateurs, Glenn and Marth Vargas state that "the table should be oriented 80 or 100 degrees away from [the long axis of the crystal]." Although we have the utmost respect for the Vargas's and their book, use the book continually as a reference, and recommend it to every faceter we train, practical experience has shown this particular prescription to be unnecessary. For cutting procedures we had to rely primarily on our own experience and knowledge.

The next step was to calculate the largest finequality stone that could be realized from this crystal. In this case, the goal was to exceed the 560 -ct. kunzite the author had cut the previous year. Given the dimensions and the weight of this first stone, we felt that we could predict the probable weight of a second stone of the same species. The two stones would be different in shape and the new stone was to be cut with different angles, so an exact comparison was impossible; yet the possible length $\times$ width $\times$ depth of the new stone correlated to the dimensions of the first such that a 700-ct. stone was deemed possible. Using these crude mathematical calculations, we marked a line on the crystal with a diamond scribe. That line was the place to begin sawing.

The initial sawing was done by the author (figure 2) because of his greater experience with kunzite. A Raytech saw was used, with Johnson Brothers blades. As a precaution against shocking the stone, the decision was made to change the saw blades at an extremely high frequency, two per cut. Because kunzi te resists abrasion, it ruins any diamond tool, including saws. It seemed logical that a dull blade would generate a great deal of heat and possibly ruin the crystal /subsequent experiments with inferior material proved this point). The first saw cut was made starting at $3: 00$ p.m. (figure 3). Two hours and thirty-three minutes later we had a crystal section that weighed 1,298 cts. Nearly 40 minutes of this time was devoted to cooling down either the cutting oil or the crystal. We changed saw blades twice during that period.

Two and one-half hours to make a single saw cut is a very long time and demonstrates the

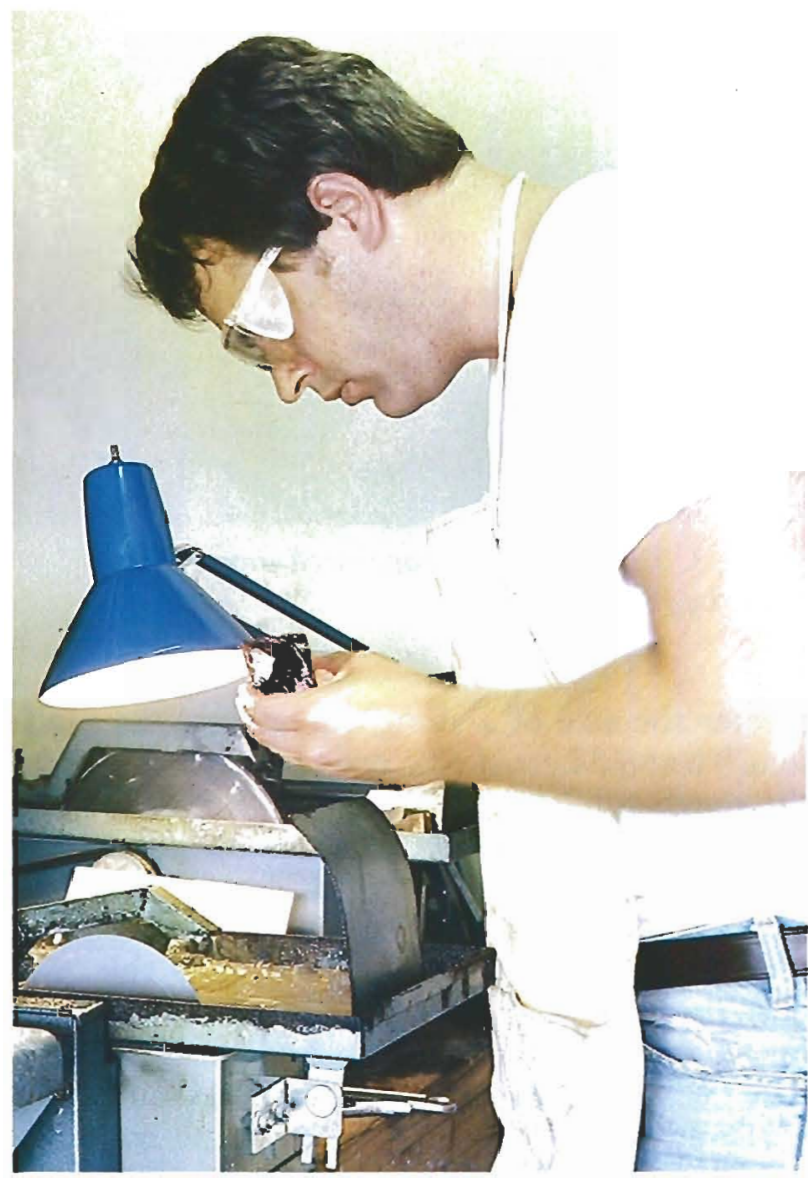

Figure 2. The author examines the crystal during the sawing process.

amount of care required for this material. Sawing a quartz crystal of equivalent dimensions would take about five minutes.

The next step in the process was to dop the 1,298 -ct. piece for faceting. Dopping involves attaching a stone for cutting to a dop stick-a metal rod that is inserted into the faceting machine. In this way the faceting machine holds the stone during cutting and permits very accurate grinding (abrading). In most faceting, the material holding the stone to the dop stick is a type of sealing wax, heated along with the stone to form the bond. In the case of extremely large stones, however, the danger of thermal (heat) shock is great because of the temperature difference between the outside of the stone, where heat is applied, and the interior of the stone, which starts out at room temperature. This is why steam cleaning is not recommended for jewelry containing kunzite. The 


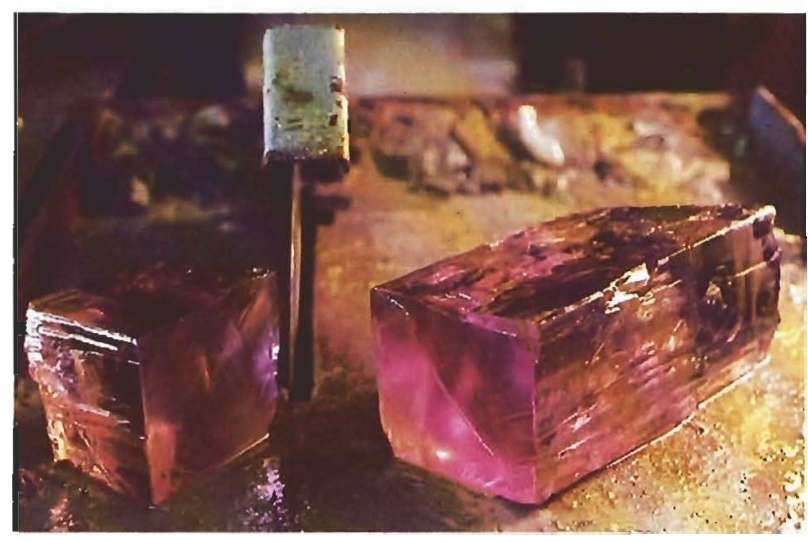

Figure 3. The crystal as it appeared moments after the first cut.

greater the size of a stone, the greater the problem. To avoid heat shock, epoxy was used to bond the rough stone to the dop stick.

One of the main concerns was whether or not the 1,298-ct. piece of rough would yield a finished stone of $700 \mathrm{cts}$. We calculated that our recovery rate had to be $54 \%$, a little on the high side. Yet both the shape and cleanliness of the rough crystal were good, and the cutter had already proved that he was capable of a good recovery rate.

Another concern was the ability to maintain cutter concentration on the project (see figure 4). The demands of this kunzite-the slow grinding process when working across the end of the crystal, the possibility of splitting, and the high value of the stone-made the job very tedious. Yet a slow, tedious procedure must be observed to avoid fracturing or cleaving the stone. Jewelers face similar problems with kunzite during the mounting of a finished stone.

The grinding process that the cutter started with is called rough cutting, which is done with a coarse-grit diamond wheel. Normally a stone of this size would be ground with a grit of 180 . Given the delicate nature of kunzite, however, a grit half that coarse, 360 , was used. The rough cutting of the pavilion took three days. By way of contrast, the entire faceting process for a 20-ct. tourmaline, aquamarine, or garnet would normally take only a few hours. The faceting machine used for the kunzite was a second-generation design by Bryant Harris of Fallbrook, California.

Fortunately, the grinding process, which was potentially the most dangerous portion of the work (i.e., when splitting was most likely to occur), went well. The next steps, fine grinding and polishing, were unlikely to cause cleavage problems and went faster than the rough grinding. When the rough grinding was completed, we felt confident that the stone would hold together for the entire process, and it did. After 14 days, Carleton finished the first stone-703.5 cts. (see figure 5). We had reached our goal and were accurate in our calculations to within $0.49 \%$ or $49 / 1,000$. More realistically, we were lucky within $99.999 \%$.

The remaining stones went smoothly and more quickly. These stones weighed 725 cts., 514 cts., and $145 \mathrm{cts}$. respectively (see figure 6). The onekilo Brazilian crystal had yielded four beautiful stones totaling 2,087 cts. Not only were the finished gems large but, as can be seen from the photographs, they were also of the finest possible color for kunzite.

Figure 4. Carleton faceting the kunzite. This custom-made faceting machine, a secondgeneration design by Bryant Harris of Fallbrook, California, is big enough to cut the world's largest faceted stone.

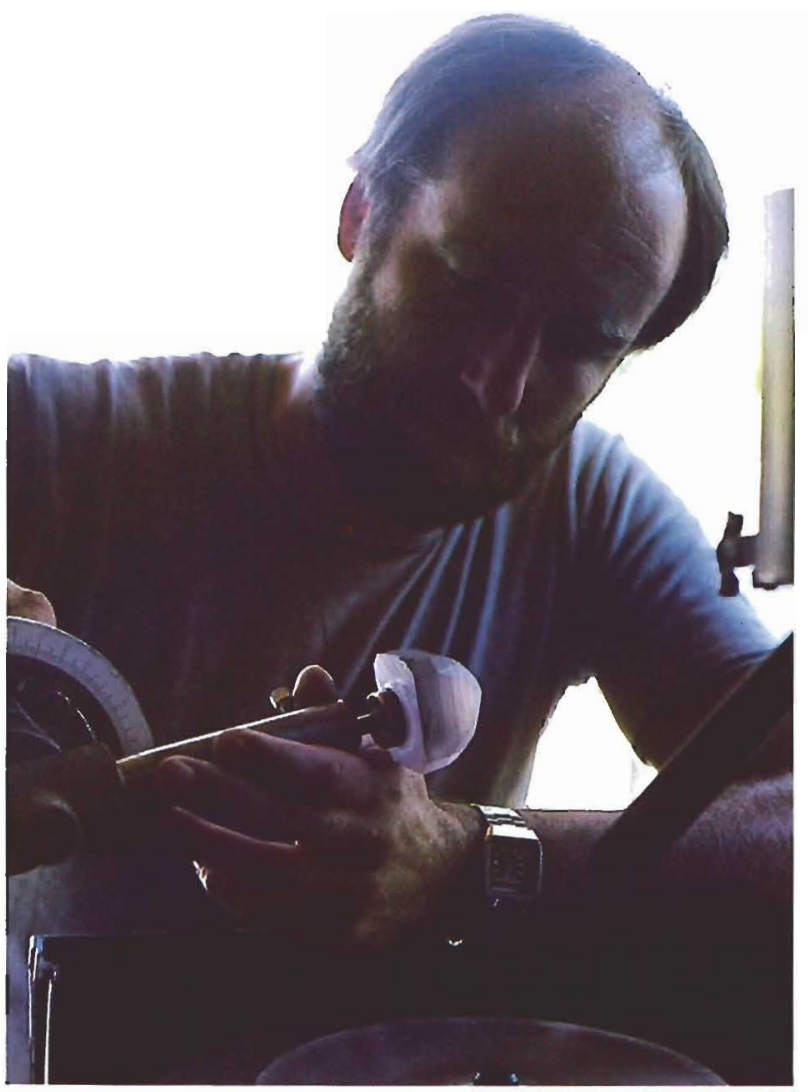


Figure 5. The first gem faceted from the crystal, $703.5 \mathrm{cts}$. The second stone is in progress, and the third still shows its crystalline origins. Photograph 1981 Laura Ramsey.
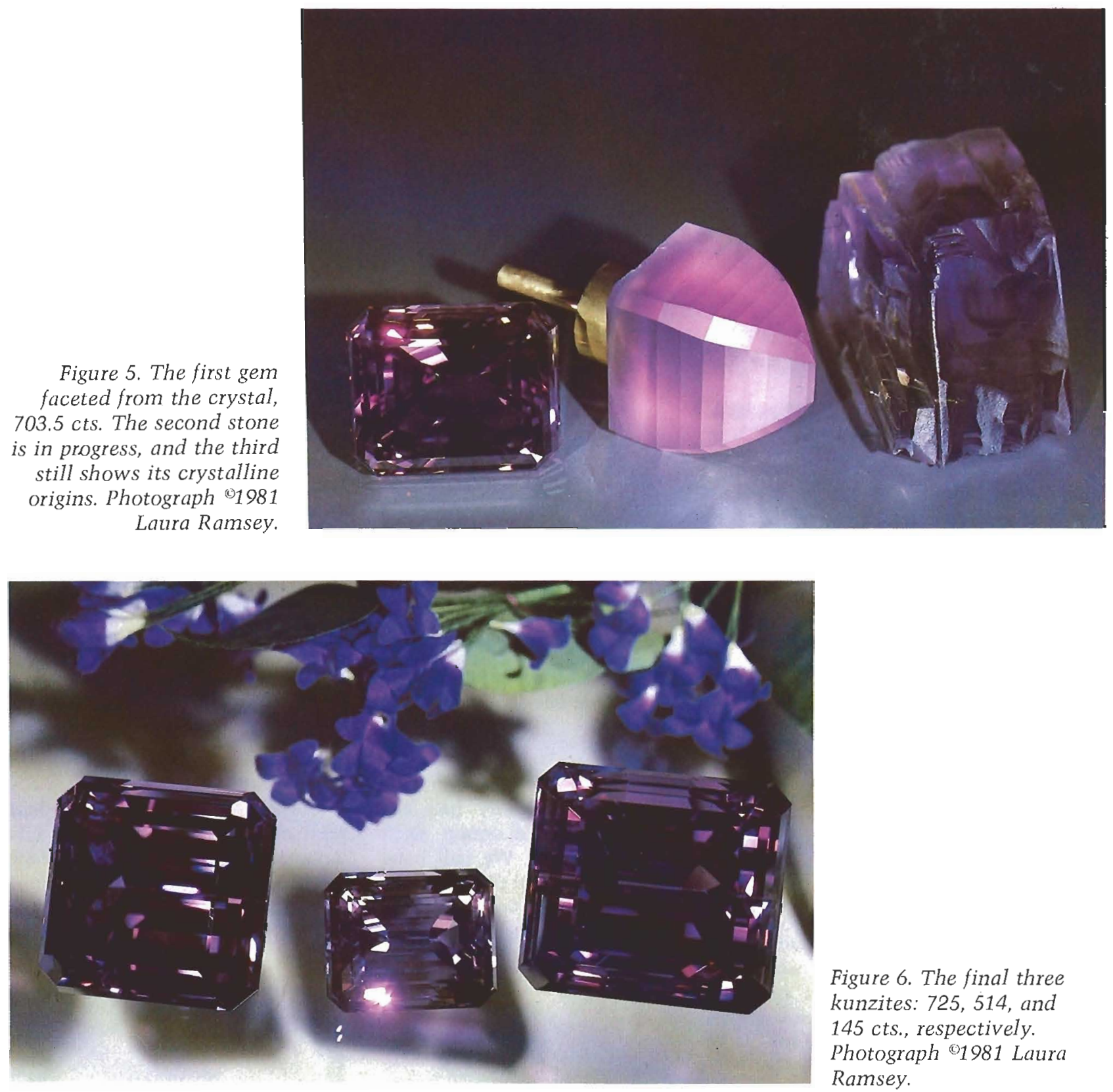

Figure 6. The final three kunzites: 725,514 , and 145 cts., respectively. Photograph 1981 Laura Ramsey.

\section{CONCLUSION}

The care shown in working this crystal into finished gems emphasizes the need for care that everyone must show kunzite. The jeweler must exercise caution in the choice of a protective mounting and in the mounting process. Proper admonition to purchasers to avoid shock to the stone is also advised. Only when jewelers and their customers are adequately educated in the properties of all colored stones will the market be able to fully utilize and appreciate these gems.

\section{REFERENCES}

Sinkankas J. (1959) 1800 sea-green carats.Gems \&) Gemology, Vol. 9, No. 10, pp. 299-305.

Vargas G., Vargas M. (1977) Faceting for Amateurs. Published by the authors, Thermal City, CA. 